\title{
An Interactive Exploration Tool for Detailed E-Vehicle Range Analysis
}

\author{
Benjamin Pichler, Andreas Riener \\ Institute for Pervasive Computing, Johannes Kepler University Linz/Austria
}

\begin{abstract}
Increasing the operating distance and, in particular, the accuracy of charge state/remaining range displays of electrical vehicles (EVs) are the most important and challenging goals to gain market penetration and customer satisfaction. In addition to environmental conditions such as outdoor temperature, precipitation, elevation profile, etc., the operating distance of an EV is dictated to a great extent by the energy consumption of electrical appliances (heating, $\mathrm{A} / \mathrm{C}$, headlamps, etc.). Range displays currently installed in electric vehicles do not reflect on that issue, predicting the remaining operating distance only based on the average total energy consumption from the past few trips. In order to increase the awareness of fleet operators and drivers on the influence of all the different range-influencing factors on the actual operating distance, we present with this project an explorative tool to analyze, visualize, and compare in detail real-world data from 1000s of tracks recorded within a car-sharing network. The main goals of this work are 1) to show and discuss the steps required to process and enhance recorded trips and 2) to highlight the power and flexibility of the exploration tool, shown on the example of the coherence between vehicle energy consumption and outdoor temperature.
\end{abstract}

\section{Keywords}

Electric vehicles, Battery drain, Range-influencing factors, Car-sharing network, Exploration tool.

\section{Introduction}

Although battery electric vehicles (BEVs) are becoming more and more popular in recent times, there are still a number of drawbacks and problems compared to traditional vehicles with combustion engines, such as long charging periods, limited range or "range anxiety" (Zimmer et al., 2011) to name a few. In addition, battery capacity is exposed to tremendous variation caused by changing environmenal conditions (Zhang \& Wang, 2009). Many others factors with potentially high impact on battery drain are not taken into account when estimating and displaying the remaining operating distance the driver. Most of the time, range is just calculated based on the average consumption over the last few trips, which is 
known to be highly inaccurate for BEVs. To overcome such issues and provide better range estimations, it is necessary to investigate on underlying problems and find out more about possible hidden coherences and dependencies. A detailed investigation of range-influencing factors is the actual focus of this work. According to previous work and our own preliminary analysis, some of the most influential factors on BEV range and efficiencey (expressed as \% battery drain per kilometer) are:

- External and environmental conditions: outdoor temperature, insulation, wind, atmospheric conditions (e.g., rain), pavement and road condition, topology (elevation profile), traffic, etc.

- Vehicle dependent factors: inside temperature, car body (air resistance), empty weight, tires (rolling resistance), battery and remaining capacity.

- Personal properties: driving style/behavior, occupants and payload, distance to vehicles in front (slipstream), plugged electric devices, settings of electric consumers like lights, A/C, heating, in-car entertainment systems, and others.

As each driven track (trip) with an electric vehicle has different characteristics regarding these parameters, it is difficult to compare real-world tracks and reason about coherences. Therefore, our intention was to develop a (web-based) tool for interactive exploration and analysis of recorded EV trips. In a first attempt, limited mainly by the available on-board unit (OBU), only a subset of all the parameters indicated above could be recorded, processed, and finally made available for analysis. In a next step, we would like to add more parameters.

\subsection{Related approaches}

Various attempts have been made with different success to measure range-limiting factors of electric vehicles (EVs) under both laboratory and real conditions. Either single components like batteries were examined (Zhang \& Wang, 2009) or the performance of vehicles as a whole was researched under varying conditions (Meyer et al., 2012). Studies have also shown wide differences between operating distances advertised by car manufacturers (measured on the basis of a standard driving cycle such as the New European Driving Cycle, NEDC) and real empirical measurements (Mock et al., 2013). Providing more accurate range information for EVs is thus essential to reduce range anxiety and customer satisfaction (and in succession, stimulate demand).

Common to all the previous projects is, that there is hardly any tool available to researchers (or interested parties like EV fleet operators or drivers) that is looking on real-world driving data to the full extent, thus incorporating all (or at least most) of the range-influencing factors, and allowing to interactively explore and compare different data sets in an easy yet intuitive way.

\section{Methodology}

With an intuitive interface for exploration and comparison of tracks with diverse characteristics, a user can understand and reason about complex data more easily and much 
quicker. To be able to provide this kind of visual data exploration/analysis tool based on stable and accurate data, the following steps were performed (tool chain; Figure 1). Detailed descriptions of the individual steps, starting with the collection of data, and continuing with preprocessing, GPS route corrections, and data enrichment are given in the following.

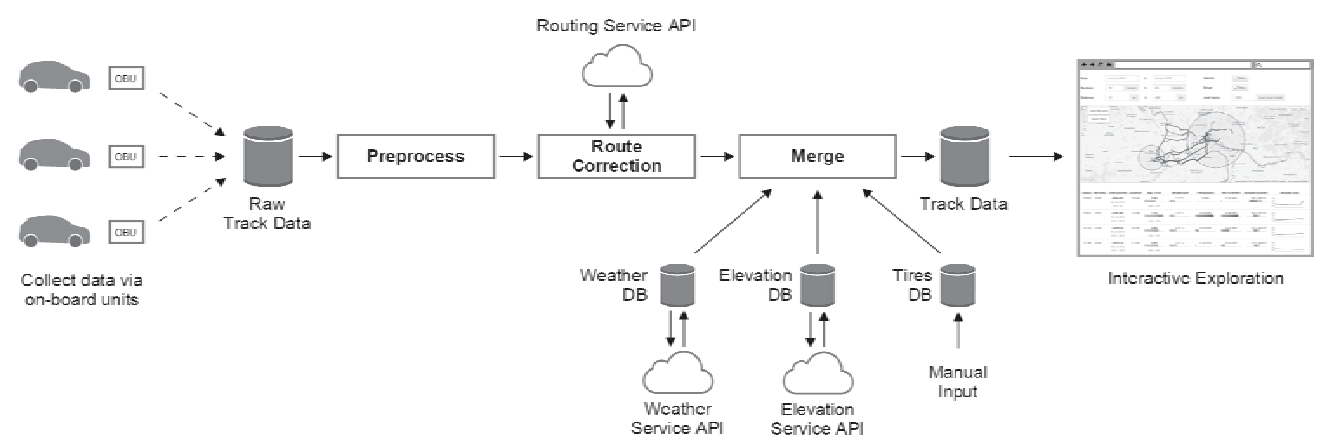

Figure 1: Processing steps executed on each EV track to be usable for later analysis and interactive exploration.

\subsection{Recording track data}

Using special on-board units (OBUs) it is possible to collect live data from a vehicle's CAN bus. Although the range of parameters is limited for most EVs, at least the charge state is available for every car. The OBU used for this project connects also to the NFC keycard reader used to lock/unlock the car, and thus allows to relate a trip to a certain driver (user ID; in the car-sharing network this project is based upon, only a few ten drivers use a particular car). Further, via an integrated GPS module, the current geolocation is captured and stored. Every few minutes (given that Internet connectivity is available), all the recorded track data are transferred to a secured SQL database.

\subsection{Processing tracks}

In a first step, raw track data retrieved from each car's on-board unit are modified and filtered in a variety of ways, based on complete tracks (trips) or selections of waypoints to filter out irrelevant tracks, e.g., based on incorrect distance or trip time information, and handle wrong or missing values in individual data sets. In the preprocessing phase we noticed that GPS coordinates of some waypoints were either not tracked detailed enough (e.g., bad GPS signal or signal reflections through buildings), or completely missing (e.g., in tunnels). Also, the sampling rate of a car's geolocation ( $30 \mathrm{sec}$.) was sometimes too low in order to calculate track information with accurate precision, thus requiring route correction.

\subsubsection{Route correction}

This step is aiming to find the exact route a car took on a track and is achieved by querying an online routing service - the "Google Maps Directions API" (Google, 2015) - and updating the actual track with precise location information. To further improve on the route 
correction, in particular for problematic parts such as points on the route mapped to the wrong, opposite lane of a road, a Divide \& Conquer-algorihm was implemented, recursively splitting a route into pieces, requesting a Google Maps route for each part, checking if distances between the original driven and the Google Maps route are already below a certain, dynamically adapted, threshold, or invoking another function call on the reduced problem size. The resulting corrected, fine-grained route is exactly matching a vehicles real driving path. Every OBU measurement (e.g., battery capacity) is in another iteration mapped back to the right position of the corrected route using a least-distance measure approach or linear mapping for regions without geolocation measurements (e.g., in tunnels).

\subsubsection{Data enrichment}

As parts of this project are based on historical driving data (starting mid 2013), some of the properties to be incorporated for detailed estimation of battery drain are not available in the track database and, thus, have to be added during (pre-)processing. Each track point is finally enriched with additional information according to the enumeration list below.

- Weather information: To access historical weather data, the online weather service "Weather Underground" (Weather Underground Inc., 2015) was queried to retrieve measurements from weather stations close-by the geolocation of route points. To reduce the number of (processing intensive) API calls, hourly measurements of temperature, precipitation, etc. are stored (cached) in a local weather database. To improve local weather accuracy, values for each waypoint on a track are determined by weighted interpolation based on distances to weather stations in the surrounding. In addition, discrete measurements are linearly interpolated regarding actual time (example: $13^{\circ} \mathrm{C}$ at $7 \mathrm{AM}$ and $14^{\circ} \mathrm{C}$ at $8 \mathrm{AM}$ finally gives $13.75^{\circ} \mathrm{C}$ at $7: 45 \mathrm{AM}$ ).

- Elevation profile: Similarly, a topology database is built-up by quering (and caching - to reduce API calls; see above) the "Google Maps Elevation API" (Google, 2015). As a result, the altitude at the geolocation of each waypoint on a track is available as additional parameter in the exploration tool (e.g., to assess the influence of recuperation on charge state).

- Tire tread pattern: Last but not least a database was created to hold tire tread patterns (mainly the rolling resistance coefficient) for all the vehicles used in the car-sharing network. The impact of summer vs. winter tires on an EVs operating distance is, according to driving tests (Kreitschmann, 2015), in the range of 5-20\%.

Due to its nature, there is no absolute need for live-recording of these information; instead, values could be retrieved via specific online services. Using these three databases, the appropriate data sets in time and space are finally merged with the correct waypoint of each track and stored in a final database queried during interactive exploration.

\subsection{The interactive exploration tool}

To visualize and analyze the tracks database, a web-based interactive user interface was developed. Its purpose is to allow a user to explore and compare tracks based on a variety of parameters. In a preselection step, tracks of interest can be selected/filtered based on parameters such as driver ID, vehicle ID, driving duration or distance. Through its interactive 
multi-view concept, the interface then offers multiple concurrent representations of the selected data within three different kinds of interconnected visualizations (Figure 2).

- List view (Figure 2A): Tracks are displayed in a tabular listing, each line representing one track. Information per track includes date/time, duration, overall distance, battery drain $(\% / \mathrm{km}$, i.e., consumed battery capacity in percent divided by the track distance), weather conditions (temperature, precipitation) and elevation. Battery drain is probably the most important measure, representing a comparable averaged measure of driving efficiency along the whole trip.

- Map view (Figure 2B): Tracks are displayed in an interactive map to easily determine their spatial properties. Routes are represented as an overlay of colorcoded polyline-graphs making use of the Google Maps API (Google, 2015).

To filter tracks according to their geolocation, one can add one or more radial filter areas within the map. This lets the user select only a subset of tracks that are (at least partly) contained in every filter area. Filtering tracks within the map view also affects the other views, i.e., subsequently showing only the remaining set of tracks.

- Visual analysis view (Figure 2C): A "parallel coordinate" visualization was implemented to transform abstract numerical track data into graphical representations using the "Data-Driven Documents" (D3.js) JavaScript library (Bostock, 2015). D3 allows the user to interactively explore high-dimensional data within a two-dimensional visualization. Each track is shown as a polyline through multiple parallel axes (columns) according to its numerical values of each parameter. This type of visualization was chosen as it allows analysis of multivariate data while at the same time offering multiple ways of interaction: axes (columns) can be reordered arbitrarily to make coherencies of values in neighboring axes more striking. And more importantly, one can define limiting ranges per axis to quickly filter tracks according to their properties. Track selections are then instantly highlighted in the parallel coordinate view as well as in the other two views.

\section{Evaluation and Discussion}

To demonstrate the core capabilities of this tool, we present and discuss in this section an exemplary analysis work-flow based on the use case of an Austrian car-sharing provider (Ibiola, 2015). The full set of tracks consists of recorded trip data from four different vehicles situated in three different areas in Austria. In total, more than 50 registered people have been driving these cars on a regularly basis from mid 2013. After pre-processing (and rejecting invalid data sets, outliers), more than 4,000 individual tracks remain for further evaluation.

Using our tool for interactive exploration allows one to set initial filters for selected track parameters: showing only tracks from one distinct vehicle (for unbiased comparisons) with a driven distance of at least five kilometers occurring in a timespan of six months (with the same set of tires mounted). This filtering results in about 400 tracks that are displayed in each of the three views. After exploring the tracks within the map view, one can draw several map filter areas to show only tracks within a certain area of interest. While the tabular list view allows already to inspect track efficiency (battery drain, $\% / \mathrm{km}$ ) under different 
conditions, one can investigate deeper working with the parallel coordinate visualization (Figure 2C). This way, it is possible to intuitively set filters for arbitrary features (axes) to immediately perceive how it affects the overall selection of tracks and corresponding parameters (on the other axes).

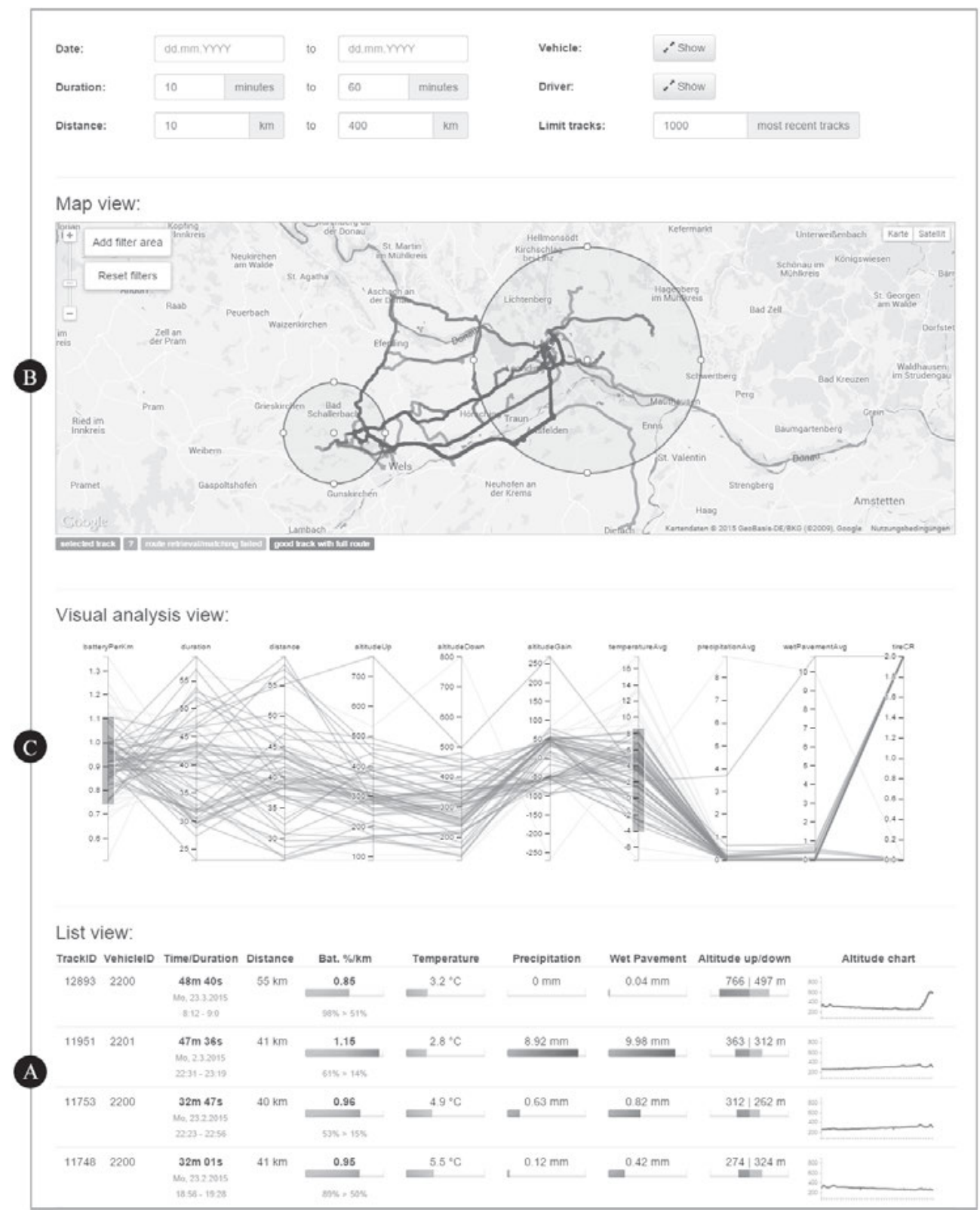

Figure 2: UI of the interactive exploration tool supporting list (A), map (B), and visual analysis $(C)$ views. 
We might, for example, limit the elevation gain to -50 to +50 meters to select only tracks driven at a rather plane surface. To check on the influence of outdoor temperature on battery drain, we can now consecutively set different temperature filters and observe the impact on the consumption scale. Figure 3 (as well as Table 1) shows this effect for two different temperature filters (cold: $+2^{\circ} \mathrm{C}$ or below; warm: +11 to $+18^{\circ} \mathrm{C}$ ).
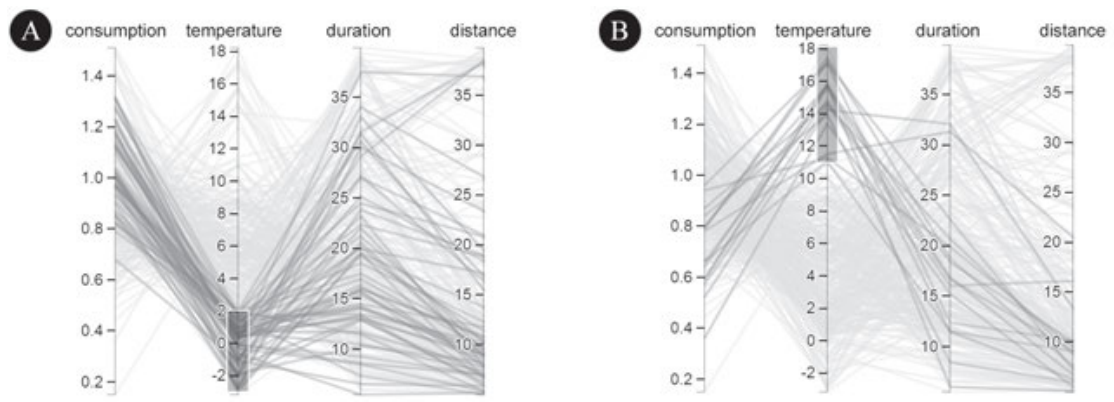

Figure 4: Parallel coordinate visualization showing coherence between outdoor temperature and battery drain. Lower temperatures on the left (A) are compared to higher temperatures on the right $(B)$ side.

\begin{tabular}{|c|c|c|c|c|c|c|c|}
\hline \multirow{2}{*}{ Selection } & \multirow{2}{*}{ Tracks } & \multicolumn{3}{|c|}{ Battery drain (\% per km) } & \multicolumn{3}{|c|}{ Outdoor temperature $\left({ }^{\circ} \mathbf{C}\right)$} \\
\cline { 3 - 8 } & & $\min$ & $\max$ & mean & min & max & mean \\
\hline A & 52 & 0.68 & 1.32 & $\mathbf{1 . 0 4}$ & -2.97 & 1.97 & $\mathbf{0 . 0 6}$ \\
\hline B & 20 & 0.36 & 0.96 & $\mathbf{0 . 7 1}$ & 11.06 & 17.44 & $\mathbf{1 4 . 6 2}$ \\
\hline
\end{tabular}

Table 1: Basic statistics corresponding to track selections $(A, B)$ as shown in Figure 3.

As expected, this kind of exploration allows us to visually detect such negative correlations of parameters in an easy and intuitive way. The coherence of outdoor temperature and battery drain might be caused by several reasons: Lower temperatures can have a negative effect on the battery capacity of BEVs (Zhang and Wang, 2009), reducing operation range and increases the average consumption. We can further assume that the heating system in the passenger cabin consumes much more energy with cold outdoor temperatures (Meyer et al., 2012) as compared to the transitional season. However, in summer time, the A/C system might again consume a lot of energy, reversing the effect for very warm or hot temperatures.

\section{Conclusion and Outlook}

In order to increase the awareness of fleet operators and drivers on the influence of different range-influencing factors on the actual battery drain or operating distance, we presented in this work an explorative tool to analyze, visualize, and compare real-world track data from a car-sharing network. The main goals of this work were to show and discuss the steps required to process and enhance recorded trips and to demonstrate the power and flexibility of the exploration tool to interactively investigate on factors influencing the range of EVs. 
Based on the existing work and the exploration tool, our perspective is to deploy a novel incar dashboard to provide a more accurate range estimation based on historical data with similar characteristics and thus to increase driver satisfaction. In addition, it is planned to include kind of gamification concepts to help drivers to further reduce battery drain and increase operating distance.

\section{Acknowledgements}

We would like to thank IBIOLA Mobility Solutions GmbH (Ibiola, 2015) for access to both the car-sharing network database and real-time data as well as for their continuous support.

\section{References}

Bostock, M. (2015). Data-Driven Documents. [Online]. http://d3js.org/. Last accessed June 28, 2015.

Google (2015). Google Maps API. [Online]. https://developers.google.com/maps/. Updated June 24, 2015. Last accessed June 29, 2015.

Ibiola (2015). IBIOLA Mobility Solutions GmbH (IMS): Smart solutions for your mobility. [Online]. http://www.ibiola-mobility.com/en. Last accessed June 29, 2015.

Kreitschmann, M. (2015). Mehrverbrauch mit Winterreifen laut Spritmonitor. [Online]. http://www.priusfreunde.de/portal/index.php?option=com_kunena\&Itemid=117\&func=view\&catid $=5 \& \mathrm{id}=281542$, March 14, 2015. Last accessed June 30, 2015.

Meyer, N., Whittal I., Christenson, M. \& Loiselle-Lapointe, A. (2012). The impact of the driving cycle and climate on electrical consumption and range of fully electric passenger vehicles. Proceedings of the International Battery, Hybrid and Fuel Cell Electric Vehicle Symposium (EVS26), Los Angeles, CA, USA, May 6-9, 2012.

Mock, P., German, J., Badnivadekar, A., Riemersma, I., Ligterink, N. \& Lambrecht, U. (2013). From laboratory to road: A comparison of official and 'real world' fuel consumption and CO2 values for cars in Europe and the United States. ICCT white paper. pp. 88. May 2013.

Weather Underground Inc. (2015). A Weather API Designed for Developers. [Online]. http://www.wunderground.com/weather/api. Last accessed June 29, 2015.

Zhang, Y. \& Wang, C.-Y. (2009). Cycle-Life Characterization of Automotive Lithium-Ion Batteries with LiNiO2 Cathode. Journal of the Electrochemical Society (ECS), 156/7, pp. A527-A535.

Zimmer, W., Buchert, M., Dittrich, S., Hacker, F., et al. (2011). OPTUM: Optimierung der Umweltentlastungspotenziale von Elektrofahrzeugen: Integrierte Betrachtung von Fahrzeugnutzung und Energiewirtschaft. Institut für Sozial-Ökologische Forschung. Berlin. 39 S.

\section{Kontaktinformationen}

Benjamin Pichler

Email: benipichler@gmail.com

Dr. Andreas Riener

Email: riener@pervasive.jku.at 